\title{
Modeling and Simulation of Hydro Mechanical Stepless Transmission Section Changing Process based on AMESim
}

\author{
Xiying Wang \\ Harbin University, 150000, China
}

Keywords: Hydraulic Machinery, Variable Speed Transmission, Mathematical Model, Section Changing Process, Ame Sim, Simulation

\begin{abstract}
Based on the study of the process of hydro mechanical CVT, the mathematical model and the convergence system of the hydraulic and mechanical circuits are established. Based on the software platform of amsion, a simulation model of hydro mechanical stepless transmission system is built. The simulation model of oil pressure circuit is constructed and simulated. Based on the analysis of the simulation results, the influence of the main parameters of the system on the process of district change is studied to improve the quality of district change and provide the basis for the formulation of the change strategy.
\end{abstract}

\section{Introduction}

AMESim is the main software for modeling, simulation and dynamic analysis of fluid machinery and transmission system developed by imagen, France[1]. It can complete complex system modeling including many fields, and provide system engineering design platform for users to perform simulation and detailed analysis. AMESim, MATALB / Simulink, Adams and other software have complete interface functions, which can learn from each other and solve many simulation and control problems of complex dynamic system. Many researchers at home and abroad use this software to solve many engineering problems. Then, three projects are applied in the research: the hydraulic system modeling and mechanism of the starting device of the excavator in Medea environment, the dynamic simulation of the hydraulic system and the two-dimensional dynamic simulation of the realization mechanism. PID controller is designed and the mining function is carried out. According to the construction of semi-active suspension (SAS) and electric power steering (EPS), the integrated control mode of saps + EPS is constructed. Matlab / Simulink and AMESim are used to realize the two combined s functions. AMESim is used to establish the engine mount simulation model. The fuzzy control method in simuliuk toolbox is used to control the engine vibration, and the semi-active control of the engine hood is simulated by the inverted model simulation. In this paper, the simulation model of the step-by-step evaluation system of hydraulic machinery is constructed in AMESim environment. The simulation model of hydraulic circuit is established by Matlab / Simulink, and the simulation is carried out by using the interface settings[2]. The influence of the main parameters of the system on the process of fragment change was investigated. It provides the basis for the quality of the segment and the strategic development of the segment.

\section{Composition and Working Principle of Hydro Mechanical Stepless Transmission System}

\subsection{Composition of Hydro Mechanical Stepless Transmission System}

The hydro mechanical CVT system is composed of oil pressure speed adjusting mechanism, mechanical speed changing mechanism, branch and convergence mechanism. Oil pressure speed adjusting mechanism and mechanical transmission mechanism are driven in parallel. The distribution of mechanical and oil pressure circuit is adjusted by branch and convergence mechanism to make the oil pressure integrated[3]. The excellent characteristics of transmission and mechanical transmission discard their own shortcomings. High transmission efficiency is achieved 
through mechanical transmission, and no power failure rate change is achieved through the combination of hydraulic transmission and mechanical transmission. Because of its inherent excellent performance, the step measurement and control system of hydraulic machinery has a broad application prospect in high-power vehicles, construction machinery and tractors.

\subsection{Section Changing Principle of Hydro Mechanical Stepless Transmission}

The output speed and the displacement of the oil pressure component of the hydro mechanical CVT change continuously and monotonously in the range of its value[4]. This process is called 1 stroke of oil pressure component, and the corresponding system output speed range is called "segment". Due to the limitation of structure, the speed adjustment range of each section of hydraulic mechanical CVT is small. In order to meet the requirements of vehicle use, the speed adjustment range is usually extended through multi-stage connection. This makes it necessary to consider switching between fragments. Section change is mainly realized by adjusting and separating the combination of section change mechanism when the transmission ratio of oil pressure circuit is adjusted to an appropriate value. In order to achieve good section change, it is necessary to select the appropriate change point and control the switching timing of the change mechanism properly. Corresponding to the oil pressure CVT system, the switching sequence of the section changing mechanism is shown in the table at the bottom corner[5]. Through the switching of the brake parts B 3 and B 2, the separation and combination switching of the oil pressure part and the oil pressure mechanical part, the brake B 3 and the gear sleeve $C 1$ are realized. Achieve high and low gear. For the sake of simplicity and explanation, only the low-frequency oil pressure section and the section change processing of the oil pressure section are analyzed[6]. According to the combination and separation of timing control elements and transmission diagram, considering the theoretical requirements of speed continuity in the transformation, the theoretical transformation point is determined as follows.

Hydraulic section system output speed $n_{b h}=n_{p c 1}=\frac{n_{s 1}}{1+k_{1}}=\frac{n_{e} \varepsilon \eta_{v}}{\left(1+k_{1}\right) i_{p}}$.

$n_{b h}$ Output speed for hydraulic section system, rad / $s ; n_{e}$ Output speed for hydraulic section system, rad / $s ; \varepsilon$ Output volume; $i_{p}$ Is the front rotation ratio of the hydraulic circuit; $\eta_{v}$ Volume efficiency of hydraulic circuit; $k_{i}$ Characteristic parameters of each planet row; $p$ Planetary gear; $p c$ Planet carrier; $s$ For the sun wheel; $r$ Gear ring; $i$ Is the corresponding component number; $n_{i}$ Is the speed of the corresponding component, $\mathrm{rad} / \mathrm{s}$.

Output speed of hydraulic mechanical section $n_{b h m}=\frac{n_{e}}{k_{2}}\left(\frac{1+k_{2}}{\left(1+k_{3}\right) i_{1}}-\frac{\varepsilon \eta_{v}}{i_{p}}\right)$.

\section{Mathematical Model of Hydro Mechanical Stepless Transmission}

\subsection{Mathematical Model of Hydraulic Speed Control System}

In modeling, the internal leakage of hydraulic components ignores the pressure loss of highpressure and low-pressure oil circuits, and assumes laminar flow, regardless of the impact of flow pulsation on on the motion characteristics of the system[7]. Establishment of high pressure oil flow equation and force balance equation of oil pressure transmission system composed of variable displacement oil pressure parts and fixed capacity oil pressure parts.

\subsection{Mathematical Model of Mechanical Circuit and Distribution and Confluence Mechanism}

In order to simplify the model, only the moment of inertia of components and loads is considered, and the effects of elasticity and attenuation are ignored. The dynamic model of each component is established to remove the internal force and the interaction force between the components of the planetary array[8]. On the basis of the restriction of the dynamic differential equation corresponding to the general formula of the dynamic differential equation of the sub convergence mechanism and the mechanical circuit under different working conditions, the mathematical model of the frequency 
range change process is established, and the dynamic differential equation under various working conditions can be established according to the combination.

$$
\begin{aligned}
& \frac{d w_{L}}{d t}=-\frac{2 k_{2}}{I_{r 2}\left(1+k_{2}\right)}\left[I_{s 3} \frac{d w_{s 3}}{d t}+I_{r 3} \frac{d w_{r 3}}{d t}-\frac{1+k_{3}}{2} T_{s 3}-\right. \\
& \frac{1+k_{3}}{2} \frac{T_{B 2}}{k_{3}}+\frac{1+k_{2}}{2} T_{M} I_{m} \frac{d w_{M}}{d t}+I_{r l} \frac{d w_{r l}}{d t}+ \\
& \left.\frac{1+k_{2}}{2} \frac{T_{L}}{k_{2}}-\frac{\left(1+k_{1}+k_{2}\right.}{2 k_{1} k_{2}} T_{B 1}\right]
\end{aligned}
$$

\section{Establishment and Simulation of Joint Simulation Model of Hydro Mechanical Stepless Transmission}

Provide users with the graphical modeling method of AMESim software. Graphic model is to model the traditional dynamic method, modularize the components, and verify them by experts in the industry. It frees users from boring mathematical modeling and focuses on physical systems. Design itself. Modeling language is an engineering language. The extension or change of the simulation model is carried out through the graphical user interface (GUI).

\subsection{Joint Simulation Model of Hydro Mechanical Stepless Transmission System}

The simplified diagram of the hydraulic pressure machine dance replies transmission system AMESim software's basic component library, the hydraulic pressure machine dance replies simultaneous interpreting system's simulation model, in order to establish the parameters used in each part, theoretical calculation and structural design, according to the set. In order to facilitate the analysis, the brake oil pressure operating system is replaced by a signal using the brake model of the component library.

For the sake of simplicity, the output of $\mathrm{K} 1$ between the mass of the vehicle's traveling star carrier and the main reducer and the reducer to the traveling star train is equivalent inertia, and the wheel's ground force and friction torque, air resistance torque to the traveling star train of $K 1$. The output of the satellite carrier is simplified as an equivalent load, which is represented by inertia and friction rotating load elements.

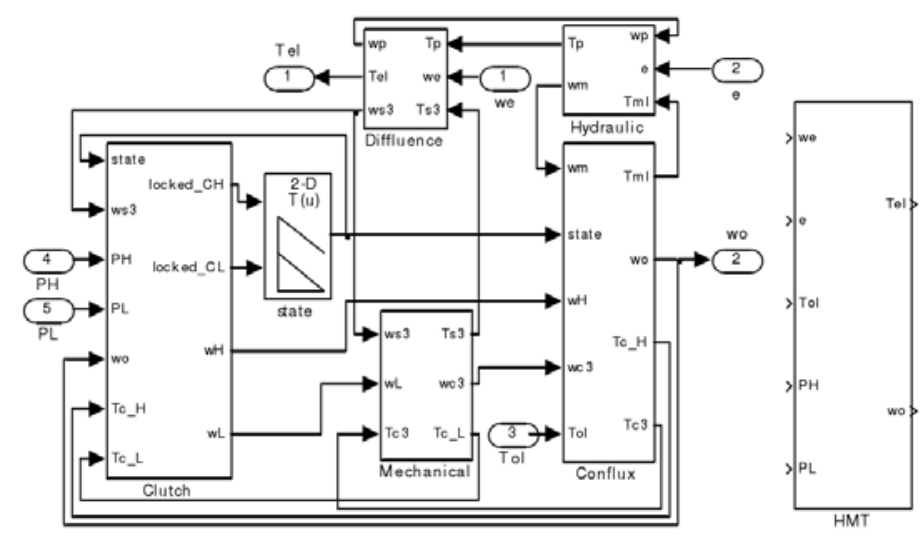

Figure 1 Simulation model of hydro mechanical stepless transmission

\subsection{Establishment of Pump Motor System Simulation Model}

AMESim software provides users with rich graphic models of basic components, which can meet the requirements of general modeling and simulation. However, according to the basic component library provided by the software, the necessity of detailed research can not meet the software itself or other software use, building an independent component model, the use of their interfaces is necessary to implement common simulation[9]. The motor system of oil pressure pump is a very complex nonlinear system. Including water leakage, friction, attenuation, liquid compression and many other factors, it plays a decisive role in the dynamic response characteristics 
of hydro mechanical stepless transmission system. There are two types of hydraulic pump and motor models in AMESim component library: one is an ideal hydraulic pump or motor model without efficiency; the other is an efficient hydraulic pump or motor model. Efficiency (including volumetric efficiency and mechanical efficiency) is the difference of constant value or displacement, rather than reflecting the motion characteristics of pump motor system under various operating conditions. For example, the use of more basic components to establish oil pump and motor model, the workload is large, various parameter settings are difficult to reflect the actual situation. Therefore, AMESim software itself can not be used to study the dynamic characteristics of CVT. According to the joint simulation model of AMSIM and Simulink, it is necessary to set up the necessary conditions and use the mathematical model of oil pressure circuit to build the pump under the MATLAB / Simulink simulation model of motor system.

\subsection{Simulation of Section Changing Process}

After setting the parameters and setting the simulation interface, you can use MATLAB / Simulink or AMESim to simulate. According to different research purposes, the research parameters are selected, and the influence of parameters on the research object is studied by batch processing function of AMESim. In this paper, the batch processing function is used to simulate the changes of load torque, load inertia, oil processing amount, brake combination, separation timing and simulation point.

\section{Analysis of Simulation Results}

The greater the load torque, the greater the change of the system output speed, the greater the impact of the change, the worse the quality of the change. However, the load torque is determined by road conditions and vehicle mass. Impact on transformation quality

Here, it is assumed that there is no overlap and interval between the separation and combination of the brakes, the variable element changes the segment with different modification ratio, and the modification rate affects the curve to change the segment change processing. It can be seen from the figure that the displacement ratio is the theoretical synchronous displacement point, the output speed changes little, the influence of displacement decreases, and the displacement quality becomes better.

The characteristics of system output speed change in program segment change processing include short time interval, synchronization and short time overlap. As can be seen from the figure, when the brake is disengaged and coupled due to a short time interval, the output speed is greatly reduced. Moreover, switching has a great impact. Moreover, the quality of exchange is poor. Also, that is considered as a segment change strategy to be avoided as much as possible. When the load torque TB is $1400 \mathrm{n} \cdot \mathrm{m}$, change the inertia influence curve of the load. It can be seen from the figure that the larger the load inertia is, the smaller the change of output speed is, and the smaller the section impact is, and the larger the dynamic load caused by the load inertia is, thus causing a certain impact on the life of system components. From the curve of the influence on the change process of the section, it can be seen that the larger the oil volume is, the greater the change of the system output speed is, and the greater the change of the section is. Therefore, it is necessary to reduce the volume of oil pressure pipeline as much as possible. The system can greatly reduce the amount of oil.

\section{Conclusion}

Based on the study of the process of hydro mechanical CVT, the mathematical model and simulation model of the stage analysis process are established, which provides the theoretical basis for the model analysis. The main influence factors of the stage change process on the AMESim badge processing function parameters using the frequency band change process influence the theoretical standards and the rule change strategy to provide reference for the formalization and improve the quality of the cutter head change. 


\section{References}

[1] Yuhanes, Dedy, Setiawan., Mehdi, Roozegar., Ting, Zou. A Mathematical Model of MultiSpeed Transmissions in Electric Vehicles in the Presence of Gear-Shifting. IEEE Transactions on Vehicular Technology, vol. XX, no. 1, pp. 1-1, 2017.

[2] Fei, Wang., Yu, Wang., Jia-hua, Han,. Experimental and simulated studies on hydraulic buffering valve for ZF-4WG308 power-shift transmission. Journal of Central South University, vol. 24, no. 8, pp. 1801-1807, 2017.

[3] Lei, Zhang., David, G. Regan, Eric P.F. Chow,. Neisseria gonorrhoeae Transmission Among Men Who Have Sex With Men: An Anatomical Site-Specific Mathematical Model Evaluating the Potential Preventive Impact of Mouthwash. Sexually Transmitted Diseases, vol. 44, no. 10, pp. 1, 2017.

[4] Dario, Rotella., Marco, Cammalleri. Direct analysis of power-split CVTs: A unified method. Mechanism \& Machine Theory, no. 121, pp. 116-127, 2018.

[5] Nargesalsadat, Dorratoltaj., Ryan, Nikin-Beers., Stanca, M. Ciupe,. Multi-scale immunoepid emiological modeling of within-host and betweenhost HIV dynamics: Systematic review of mathematical models. Peerj, vol. 5, no. 5758, pp. e3877, 2017.

[6] Meng, Li., Yong, Zhang., Fengyu, Liu.,. Manual Transmission Gear Rattle Vibration Research Based on Mathematical and Multi-Body Dynamics Co-simulation and Experimen. Journal of Shanghai Jiaotong University (Science), vol. 24, no. 4, pp. 524-533, 2019.

[7] S, Mandol., P.K.Dan., M.K Mondal. Deriving optimal transmission architecture of planetary gear assembly by mathematical modelling of design parameters. Iop Conference, vol. 393, no. 1, pp. 012062, 2018.

[8] M. Domenech, de. Cellès, A. A. King, P. Rohani. Commentary: resolving pertussis resurgence and vaccine immunity using mathematical transmission models. Human Vaccines and Immunotherapeutics, 2018.

[9] M. Domenech de Cellès, A. A. King, P. Rohani. Commentary: resolving pertussis resurgence and vaccine immunity using mathematical transmission models. Human Vaccines and Immunotherapeutics, 2018. 